\title{
ALTHOCHDEUTSCHE DIALEKTE - NEUHOCHDEUTSCHE DIALEKTE. TERMINOLOGISCHE PROBLEME BEI BESCHREIBUNGEN ÄLTERER SPRACHZUSTÄNDE
}

\begin{abstract}
AвSTRact: Old High German Dialects - New High German Dialects. Terminological problems of the description of older stages of natural languages

In the history of the German language of the period between 750-1050, the term "Old High German dialects" is used to characterize the Franconian, Bavarian and Alemannic languages, in which the records from this period are written. The author of the article analyses the meaning of the term in question and explains why the languages of those records have been called "dialects" (despite the fact that the common language, whose dialects they might have been, did not exist at that time yet), and on what grounds they have been called "German" dialects (though the German nationality did not exist at that time either).
\end{abstract}

KEYWORDS: history of language; dialectology

In der deutschen Sprachgeschichte gebraucht man für die überlieferten Sprachformen aus der Zeit zwischen 750 und 1050 den zusammenfassenden Begriff ,althochdeutsch“. Aber Überlieferungen aus der Zeit, auf welche sich diese Benennung bezieht, sind Überlieferungen in bairischer, alemannischer und fränkischer Sprache. „Es gibt keine allen gemeinsame althochdeutsche Schriftsprache, sondern nur althochdeutsche Dialekte, und jeder uns überlieferte althochdeutsche Text trägt seine besonderen Dialektmerkmale", schreibt Hans Eggers in seiner Sprachgeschichte (Eggers 1963: 39). Auch der Begriff „,deutsch“ wird in Bezug auf diese Zeit anders aufgefasst:

Deutsch als Sprachbegriff meint geschichtlich gesehen zunächst erst das langsame Zusammenwachsen der verschiedenen Stammesdialekte der fränkischen Rheinlande, des alemannischen Ober- und Hochrheingebietes, des bairischen und z. T. auch österreichischen Donau- und Alpenraumes und, durch den Alpenkamm getrennt, der langobardischen Sprachresten in Oberitalien, zu einer neuen Einheit. (Sonderegger 2003: 28) ${ }^{1}$

\footnotetext{
Norbert Morciniec - Wyższa Szkoła Filologiczna, Wrocław, n.morciniec@wsf.edu.pl

${ }^{1}$ Vgl. auch: Morciniec 2015: 34f.
} 
Was in dieser und ähnlichen Formulierungen der deutschen Sprachgeschichte zunächst auffällt, ist der Terminus „Dialekt“, der hier offensichtlich in einer anderen Bedeutung gebraucht wird als in der modernen Dialektologie. In der modernen Dialektologie setzt der Begriff „Dialekt“ eine über den Mundarten bestehende Einheitssprache voraus. Deutsche Dialekte sind Dialekte der deutschen Sprache, englische Dialekte - Dialekte der englischen Sprache usw. Althochdeutsche Dialekte wären demnach Dialekte der althochdeutschen Sprache. Aber solch eine Sprache hat es nie gegeben (Braune/Reiffenstein 2004: 1). Offensichtlich versteht man in der Sprachgeschichte Dialekte im genetischen Sinne, als verwandte sprachliche Erscheinungsformen, die sich aus einer für diese Sprache gemeinsamen Ausgangssprache (Ursprache) entwickelt haben, wobei, wie wir noch sehen werden, implizit noch zusätzliche nicht immer formulierte Kriterien eine Rolle spielen. Wollte man moderne Dialekte nur aufgrund der Sprachverwandtschaft definieren und das Kriterium der sie überdachenden Einheitssprache unberücksichtigt lassen, so gerieten wir in Widerspruch zu allgemein anerkannten Erkenntnissen. Es gibt keine scharfen Scheidungslinien, z. B. zwischen den Dialekten Nordwestdeutschlands und den östlichen Dialekten der Niederlande, den Dialekten Südpolens und der nördlichen Slowakei. Diese Dialekte sind jeweils so nahe miteinander verwandt, dass sich die Dialektsprecher diesseits und jenseits der Staatsgrenzen ohne Weiteres verständigen können. Dass die Dialekte hüben und drüben als Mundarten zweier verschiedener Sprachen anerkannt werden, beruht einzig auf der Tatsache, dass sie jeweils im Geltungsbereich einer anderen Kultursprache liegen, also von anderen Kultursprachen überdacht werden. Aber auch das Überdachungsmoment allein reicht nicht aus, um zwei Mundarten einer Sprache zuzuordnen. Das Sorbische in der Lausitz zählt man nicht zu den deutschen Dialekten, obwohl es von der deutschen Kultursprache überdacht wird.

Ähnliche Schwierigkeiten bestehen auch bei Anwendung nur eines der genannten Kriterien in Bezug auf die Zuordnung der Dialekte in älteren Sprachperioden, etwa in althochdeutscher oder mittelhochdeutscher Zeit. Da es in dieser Zeit noch keine allgemeine Einheitssprache gab, kann es sich hier wohl nur um das Kriterium Sprachverwandtschaft handeln. Aber da entsteht sofort die Frage nach dem Grad der Sprachverwandtschaft, den Dialekte aufweisen müssen, um als deutsche Dialekte bezeichnet zu werden.

Trotz dieser Bedenken wurde in der philologischen Praxis der deutschen Germanistik das Kriterium der sprachlichen Verwandtschaft weitgehend gehandhabt. Dass für die alt- und mittelhochdeutsche Zeit auch das Niederdeutsche zu den deutschen Dialekten gezählt wird, weist darauf hin, dass der Grad der Verwandtschaft ziemlich weit aufgefasst wurde. Die Mundarten auf den Britischen Inseln aber, deren Verwandtschaft mit den hochdeutschen Dialekten genetisch genommen nicht geringer ist als die des Niederdeutschen, gehören nicht mehr zu den deutschen Dialekten. Hier spricht man von alt- bzw. mittelenglischen Dialekten. Diese Tatsache kann doch nur bedeuten, dass hier stillschweigend ein zusätzliches geografisch-politisches Kriterium angenommen 
wurde, etwa „auf dem Territorium eines Staates“. Althochdeutsche Dialekte wären nach dieser Auffassung diejenigen germanischen Territorialsprachen, die auf dem Gebiete des Karolingerreiches und des späteren Deutschen Reiches gesprochen wurden. Solch eine Auffassung könnte verständlich machen, dass auch das Altsächsische und die späteren mittelniederdeutschen Mundarten als deutsche Dialekte bezeichnet werden. Allerdings müssten dann konsequenterweise zu den deutschen Dialekten auch die mittelniederländischen Mundarten gerechnet werden, da ihre Gebiete (allerdings außer Flandern) bis zum Westfälischen Frieden (1648) zum Deutschen Reich gehörten. Dieser Standpunkt wird in der Tat von den meisten deutschen Sprachhistorikern ebenso eindeutig vertreten, wie er von den niederländischen Sprachhistorikern entschlossen zurückgewiesen wird (Morciniec 2002: 83-91).

Die Koppelung der Kriterien ,,sprachliche Verwandtschaft“ mit dem geografisch-politischen Kriterium führt jedoch zu Konsequenzen, die es ratsam erscheinen lassen, diesen Faktor in sprachgeschichtlichen Erwägungen nicht zu berücksichtigen. Es ist allgemein bekannt, dass Staatsgrenzen sich im Laufe der Geschichte wesentlich verschieben können. Sollte dann ein deutscher Dialekt, der sich außerhalb der deutschen Staatsgrenzen befände, aufhören, ein deutscher Dialekt zu sein? Wir sehen: Weder das Kriterium ,sprachliche Verwandtschaft“ allein, noch seine Kombinierung mit dem geografisch-politischen Faktor, führt zu befriedigenden Ergebnissen.

Greifen wir zu den oben genannten Kriterien ,sprachliche Verwandtschaft + überdachende Einheitssprache" zurück, so wären für den Zeitabschnitt, in dem es noch keine deutsche Einheitssprache gab, folgende Möglichkeiten zu erwägen: ${ }^{2}$

\section{1.}

Als deutsche Dialekte könnten verwandte westgermanische Sprachformen angesehen werden, die von einer deutschen Einheitssprache überdacht werden, die zwar noch nicht als allgemein anerkannte Einheitssprache vorhanden ist, zu der aber bereits erste Ansätze bestehen. Erste Ansätze zu einer deutschen Einheitssprache lassen sich aber erst gegen Ende des 11. Jahrhunderts nachweisen, und so wären nach dieser Auffassung zwar die mittelhochdeutschen Mundarten schon deutsche Dialekte, das Althochdeutsche dagegen könnte noch nicht als Deutsch bezeichnet werden. Aus dieser Auffassung ergäben sich aber auch noch weitere die mittelhochdeutsche Zeit betreffende Konsequenzen. Da es seit dem 13. Jahrhundert auf niederdeutschem Sprachgebiet Ansätze zu einer niederdeutschen Schriftsprache gab, wären auch die mittelniederdeutschen Mundarten (noch) keine deutschen Dialekte. Diese mittelniederdeutsche Schrift- und Verkehrssprache wurde seit dem 14. Jahrhundert im Geltungsbereich der Hanse zur Ver-

\footnotetext{
${ }^{2}$ Wir übernehmen hier mutatis mutandis einige von Jan Goossens für das Mittelniederländische geltende Erwägungen (Willemyns 1979: 3-17).
} 
kehrssprache des gesamten europäischen Nordens. Seit dem Niedergang der Hanse und dem Vordringen der Reformation beginnt die hochdeutsche Schriftsprache sich auch in Norddeutschland durchzusetzen. Erst jetzt, also seit der ersten Hälfte des 16. Jahrhunderts, könnten die niederdeutschen Mundarten als deutsche Dialekte angesehen werden.

Ähnliches gilt mutatis mutandis auch für die mittelniederländischen Mundarten. Da sich auf südniederländischem Gebiet seit dem 13. Jahrhundert eine niederländische Schriftsprache entwickelt hatte, können diese Mundarten nicht als deutsche, sondern nur als niederländische Dialekte bezeichnet werden. Anders als in Norddeutschland hat die hochdeutsche Sprache auf niederländischem Gebiet niemals Fuß fassen können. Im Gegenteil: Hier hat ein im nationalen Aufstand gegen die spanische Herrschaft sich festigendes Sprachbewusstsein zur Ausbildung der niederländischen Hochsprache geführt, die als überdachende Sprachform über allen niederländischen Dialekten zu fungieren begann.

\section{2.}

Als zweite zu erwägende Möglichkeit bietet sich folgender Gedankengang an: Als deutsche Dialekte könnten verwandte westgermanische Sprachformen angesehen werden, in denen Merkmale auftreten, die in späterer Zeit in der modernen deutschen Kultursprache als exklusive Merkmale wiederzufinden sind, das heißt als solche Merkmale, die nur der deutschen Hochsprache eigen sind und in keiner anderen germanischen Kultursprache vorkommen. Diese Auffassung scheint auf den ersten Blick für sprachhistorische Erwägungen recht attraktiv und brauchbar zu sein. Exklusive Merkmale lassen sich nämlich schon in den ältesten althochdeutschen Sprachdenkmälern feststellen, und somit könnten die Sprachen dieser Denkmäler mit Recht als deutsche Dialekte bezeichnet werden. Aber auch diese Auffassung bringt einige nicht zu übersehende Schwierigkeiten mit sich. Wie viele für das spätere Deutsch exklusive Merkmale müsste ein Sprachdenkmal aufweisen, um als ,deutsch“ bezeichnet zu werden? Genügt ein Merkmal oder müssen es mehrere sein? In der Praxis scheinen sich diese Bedenken aber weitgehend aufzulösen. Es stellt sich nämlich heraus, dass in den ahd. Sprachdenkmälern in der Regel Merkmalkomplexe vorkommen, die in der deutschen Hochsprache als charakteristische Merkmale gelten. Als solch ein Merkmalkomplex kann z. B. eine Gruppe von lautlichen Neuerungen angesehen werden, die im Ergebnis der zweiten Lautverschiebung entstanden ist. Da in der Sprache der ältesten Denkmäler aus dem Norden des heutigen deutschen Sprachgebietes, wie etwa im Heliand aus dem 9. Jahrhundert, die zweite Lautverschiebung nicht eingetreten ist, hier also die alten germanischen Konsonanten $p, t, k$ so wie in den anderen germanischen Sprachen erhalten geblieben sind, kann diese Sprache konsequenterweise nicht als deutsch bezeichnet werden. Das entspricht durchaus dem Gebrauch in den meisten deutschen Sprachgeschichten, die hier den Ausdruck ,altsächsisch“ verwenden. 
Eine weitere Konsequenz der unter Nr. 2 formulierten These ist die Tatsache, dass die Sprache mancher Sprachdenkmäler aufgrund ihrer exklusiven Merkmale, sowohl zu den deutschen Dialekten, als auch zugleich zu den Dialekten einer anderen Sprache, z. B. des Niederländischen gezählt werden kann. Das ist z. B. mit der altniederfränkischen Psalmenübersetzung, den sogenannten Wachtendonckschen Psalmen, der Fall, die wahrscheinlich im 9. Jahrhundert entstanden sind. In diesem Sprachdenkmal treten sowohl (besonders in den ersten neun Psalmen) exklusive deutsche Merkmale auf (Lautverschiebung) als auch exklusive niederländische Merkmale (wie etwa die Entwicklung von germ. $\mathrm{ft}>\mathrm{cht}$, vgl. dtsch. stiften, niederl. stichten oder von germ. hs $>$ ss, vgl. dtsch. wachsen, ndl. wassen). Dieser Befund entspricht der Tatsache, dass die Wachtendonckschen Psalmen von deutschen Sprachhistorikern zu den altdeutschen Denkmälern gezählt werden, während die niederländische Forschung sie als altniederländisches Denkmal auffasst. L. de Grauwe schreibt in seiner Ausgabe der Wachtendonckschen Psalmen (de Grauwe 1979: XXXVI) über ihre Sprache völlig mit Recht: „Onze Psalmen zijn dus deels Oudhoogduits deels Oudnederlands.“3

Alles in allem scheint das Kriterium der exklusiven Merkmale in Verbindung mit dem Merkmal „Sprachverwandtschaft“ ein recht brauchbares Kriterium zu sein, um zu einer Zeit, in der es noch keine deutsche Einheitssprache gab, die deutschen Dialekte von den nicht deutschen zu unterscheiden. Der Gebrauch dieser Kriterien führt zu Ergebnissen, die durchaus den traditionellen Erkenntnissen und Benennungen der deutschen Philologie entsprechen. Mit einer Ausnahme jedoch: Wenn manche Sprachhistoriker das Altsächsische und das Altniederfränkische unter dem Namen Altniederdeutsch zusammenfassen, so sollte dies lieber unterbleiben. Als deutsche Dialekte können das Altsächsische und das Altniederfränkische nach dem Kriterium der exklusiven Merkmale nicht bezeichnet werden.

\section{3.}

Es ließe sich noch eine dritte Möglichkeit erwägen, die es erlauben würde, auch das Altsächsische zu den (alt)deutschen Dialekten zu zählen. Als solche bietet sich etwa an: Miteinander verwandte westgermanische Dialekte, über denen in späterer Zeit die deutsche Hochsprache die Rolle einer überdachenden Sprache übernommen hatte. Solch eine Auffassung könnte gewährleisten, dass aus rein praktischen Gründen der altsächsische Heliand in einer deutschen Sprach- bzw. Literaturgeschichte mit behandelt wird.

Eine Geisteswissenschaft nämlich, die ihrem Arbeitsgebiet großzügig auch jene Grenzfälle zuzählt, die von Nachbardisziplinen vernachlässigt werden müssten, oder gar von keiner Wissenschaft behandelt werden würden, stellt dadurch sicher, dass diese überhaupt Teil eines wissenschaftlichen Aufgabenbereiches sind. (Goossens 1971: 20)

\footnotetext{
${ }^{3}$ „Unsere Psalmen sind also teils Althochdeutsch teils Altniederländisch.“
} 
Unsere bisherigen Überlegungen sollen uns nicht darüber hinwegtäuschen, dass die Wahl der Kriterien zur Bestimmung der altdeutschen Dialekte eigentlich arbiträr und vor allem unhistorisch ist in dem Sinne, dass wir als Kriterium Merkmale vom Standpunkt der heutigen deutschen Sprache ausgewählt haben, ohne die historischen Umstände zu berücksichtigen, in denen sich diese Dialekte befanden. Ein althochdeutscher Sprecher sprach seine fränkische, bairische oder alemannische Sprache, im Rahmen deren es gewiss zahlreiche lokal und sozial bestimmte Gebrauchsvarianten gab. Wenn wir aber diese Sprachen heute als althochdeutsche Dialekte bezeichnen, so verstehen wir diese Benennung eben arbiträr, vereinbarungsgemäß zum Zwecke einer wissenschaftlichen Klassifikation. Das ist ein wohlbegründetes Recht jeder Wissenschaft. Verwirrungen entstehen erst dann, wenn in der Sprachgeschichte Begriffe gebraucht werden, ohne sie zu definieren, und der nicht eingeweihte Leser sie intuitiv in ihrer umgangssprachlichen Bedeutung versteht.

\section{Literatur}

Braune, Wilhelm (2004): Althochdeutsche Grammatik. 15. Auflage, bearbeitet von Ingo Reiffenstein. Tübingen: Walter de Gruyter.

De Grauwe, Luc (1979): De Wachtendoncksche Psalmen. Gent: Koninklijke Academie voor Nederlandse Taal- en Letterkunde.

Eggers, Hans (1963): Deutsche Sprachgeschichte I. Das Althochdeutsche. Reinbeck bei Hamburg: Rowohlt. Goossens, Jan (1971): Was ist deutsch und wie verhält es sich zum Niederländischen? Bonn: Presse und Kulturabteilung der Kgl. Niederländischen Botschaft.

Sonderegger, Stefan (2003): Althochdeutsche Sprache und Literatur. 3. Auflage. Berlin/New York: Walter de Gruyter.

Morciniec, Norbert (2002): „Niederländisch und Deutsch. Zur Klärung einiger Fehldeutungen des Niederländischen in der deutschen Sprachgeschichtsschreibung. "In: Niederländische Sprach- und Literaturwissenschaft im europäischen Kontext. (Bd. 12 der Akten des X. Internationalen Germanistikkongresses Wien 2000), Bern u. a.: Peter Lang Verlag. 83-91.

Morciniec, Norbert (2015): Historia języka niemieckiego. Wrocław: Wydawnictwo Wyższej Szkoły Filologicznej we Wrocławiu.

Willemyns, Roland (1979): Het niet-literaire middelnederlands. Herausgegeben von R. Willemyns unter Mitarbeit von J. Goossens. Assen/Brügge: Van Gorcum. 